\title{
Equilíbrio em idosos institucionalizados e não institucionalizados e sua relação com a qualidade de vida
}

\author{
Balance in institutionalized and non-institutionalized elderly and their relationship with \\ quality of life
}

\author{
Hugo Hudá de Freitas ${ }^{1}$, Fábio Ricardo Acencio ${ }^{2}$, Daniel Vicentini de Oliveira ${ }^{3 *}$, \\ Sônia Maria Marques Gomes Bertolini ${ }^{4}$
}

\begin{abstract}
${ }^{1}$ Graduação em Educação Física; ${ }^{2}$ Mestre em Promoção da Saúde Universidade Cesumar; ${ }^{3}$ Doutor em Gerontologia. Pós-doutorando em Educação fisica. Universidade estadual de Maringá ; ${ }^{4}$ Doutora em ciências Morfofuncionais. Docente no programa de pós graduação stricto sensu em Promoção da saúde.
\end{abstract}

\begin{abstract}
Resumo
Introdução: o envelhecimento gera alterações no equilíbrio que podem impactar a qualidade de vida do idoso. Objetivo: avaliar o equilíbrio de idosos institucionalizados e não institucionalizados e sua relação com a qualidade de vida. Metodologia: estudo transversal, realizado com 20 idosas, das quais 10 institucionalizadas (G1) e 10 não institucionalizadas (G2). Foram aplicados a Escala Motora para Terceira Idade (EMTI) e o SF-12. Os dados foram analisados por meio dos testes Shapiro-Wilk, correlação de Pearson e t de student $(p<0,05)$. Resultados: não foi encontrada diferença significativa $(p>0,05)$ no equilíbrio entre os grupos. Observou-se que a correlação entre qualidade de vida e equilíbrio no $\mathrm{G} 1(0,757)$ é forte, ao passo que no $\mathrm{G} 2(0,448)$ é moderada $(p<0,05)$. Conclusão: não há diferença no equilíbrio entre idosos institucionalizados e não institucionalizados no estudo realizado, porém a relação entre essa variável e a qualidade de vida é significativa e forte em idosos institucionalizados, e moderada em idosos não institucionalizados. Palavras-chave: Atividade motora. Exercício. Envelhecimento.
\end{abstract}

\begin{abstract}
Introduction: aging generates changes in balance, which can impact the quality of life of the elderly. Objective: to evaluate the balance of institutionalized and non-institutionalized elderly and its relationship with quality of life. Methodology: cross-sectional study, conducted with 20 elderly women, 10 institutionalized (G1) and 10 non-institutionalized (G2). The Third Age Motor Scale (EMTI) was applied and, for quality of life, the SF-12. Data were analyzed using Shapiro-Wilk tests, Pearson correlation and student's t-test $(p<0.05)$. Results: no significant difference $(p>0.05)$ was found in the balance between the groups. The correlation between quality of life and balance in $\mathrm{G1}(0.757)$ is strong, while in $\mathrm{G} 2(0.448)$ it is moderate $(p<0.05)$. Conclusion: there is no difference in balance between institutionalized and non-institutionalized elderly in this study. However, the relationship between this variable and quality of life is significant and strong in institutionalized elderly and moderate in non-institutionalized elderly.

Keywords: Motor activity. Exercise. Aging.
\end{abstract}

\section{INTRODUÇÃO}

Dentre as habilidades alteradas com o envelhecimento, destaca-se o equilíbrio, necessário para realização de atividades básicas da vida diária. $O$ déficit dele em idosos traz diversas consequências, e uma das mais preocupantes é a queda, que pode gerar sequelas físicas, psicológicas e até levar à morte (ANTES et al., 2015).

Além de mudanças físicas, como no equilíbrio e na força muscular, o processo de envelhecer provoca diminuição da qualidade de vida, que envolve autoestima, bem-estar pessoal, capacidade funcional, nível socioeconômico, estado emocional, interação social, atividade intelectual, autocuidado, suporte familiar, estado de saúde, valores culturais, éticos e religiosos, estilo de vida, satisfação

Correspondente/Corresponding: *Daniel Vicentini de Oliveira - End: Avenida Guedner, 1610. Jardim aclimação. CEP 87050-900. Maringá, Paraná - Tel: (44) 99942-8575 - E-mail: d.vicentini@hotmail.com com o emprego e/ou com as atividades cotidianas e com o ambiente em que se vive. Dessa forma, o conceito de qualidade de vida é muito pessoal e depende diretamente do nível sociocultural, da idade e da busca por realizações pessoais de cada indivíduo (FREITAS et al., 2017; PUCCI et al., 2012).

Em virtude de baixa qualidade de vida, assim como das diversas alterações biopsicossociais dessa etapa da existência, tem aumentado a prestação de serviços de cuidados formais oferecidos por Instituições de Longa Permanência para Idosos (ILPI). São locais de caráter residencial e domicílio coletivo de pessoas com idade igual ou superior a 60 anos, possuindo ou não suporte familiar, em condições de liberdade, dignidade e cidadania, podendo estar ou não vinculados à iniciativa governamental. Idoso institucionalizado é, na maioria das vezes, aquela pessoa que foi privada de seus projetos, amigos, família e até mesmo das relações que constituem sua história (ALLENDORF et al., 2016; SILVA et al., 2015). 
Sabe-se que quanto maior for o tempo de institucionalização, mais fragilizado ficará o idoso, já que há mudança do seu ambiente, alteração da função psicológica, cognitiva e funcional, comprometendo a saúde e qualidade de vida, aumentando também os riscos de queda (FERREIRA; YOSHITOME, 2010).

Portanto, com a crescente expectativa de vida e a expansão da população idosa, especialmente daquela institucionalizada, são necessários estudos sobre qualidade de vida dela, principalmente quando relacionados às capacidades físicas como o equilíbrio. Posto isso, o objetivo deste estudo foi avaliar o equilíbrio de idosos institucionalizados e não institucionalizados e sua relação com a qualidade de vida.

\section{METODOLOGIA}

\section{Caracterização do estudo}

O presente estudo se caracteriza como analítico, observacional e transversal e foi aprovado pelo Comitê de Ética em Pesquisa por meio do parecer n. 2.776.910/2018.

\section{Participantes}

A amostra foi escolhida de forma intencional e por conveniência e composta por 20 idosas, das quais 10 residentes no Recanto dos Velhinhos, da cidade de Cianorte (PR) (G1), e 10 frequentadoras de uma academia da terceira idade (ATI) (G2).

Puderam participar da pesquisa idosas com 60 anos ou mais, que moravam na instituição ou que frequentavam a ATI. Não foram incluídos idosos do sexo masculino e os que possuíam doenças/lesões ortopédicas e/ou neurológicas que impedissem a realização dos testes de equilíbrio. Aqueles com possíveis déficits cognitivos, avaliados pelo Miniexame do Estado Mental, também foram excluídos (BRUCKI et al., 2003; FOLSTEIN; FOLSTEIN; MCHUGH, 1975).

A Escala Motora para Terceira Idade (EMTI), validada por Rosa Neto (2002), foi o instrumento utilizado para verificar o nível de equilíbrio das idosas. O protocolo consiste nos testes: equilibrar-se sobre um banco; equilibrar-se sobre um joelho; equilibrar-se com o tronco flexionado; equilibrar-se nas pontas dos pés; fazer o "pé manco"; fazer o "quatro"; equilibrar-se de cócoras; equilibrar-se com o tronco flexionado

O SF-12 consiste em 12 questões investigativas sobre percepção de qualidade vida relacionada à saúde física e mental do indivíduo. Os participantes avaliaram suas respostas em escalas de cinco pontos, de "Excelente" para "Muito ruim". A pontuação varia em uma escala de zero a 100, e os maiores escores são associados a melhores níveis de qualidade de vida (CAMELIER, 2004).

\section{Procedimentos de coleta de dados}

A coleta de dados foi realizada no período de agosto e setembro de 2018. Os testes foram aplicados no Recanto dos Velhinhos, local de morada das idosas com dia e horário previamente agendado com o responsável; a atividade foi conduzida pelos pesquisadores e acompanhada por uma enfermeira da ILPI. Com as idosas da ATI, a coleta foi feita em dias e horários diversos, na própria academia, também pela equipe de pesquisadores.

Antes de iniciar a pesquisa, foram informados às idosas os procedimentos e objetivos dela. Aquelas que aceitaram participar, assinaram um Termo de Consentimento Livre e Esclarecido (TCLE). Cada coleta durou, em média, dez minutos por idosa.

\section{Análise dos dados}

Os dados obtidos foram tratados por meio de estatística descritiva e inferencial e tabulados no programa Excel $^{\circledR}$; as variáveis descritivas foram apresentadas em forma de média \pm desvio padrão (DP). A normalidade dos dados foi verificada pelo teste de Shapiro-Wilk. A relação entre as variáveis foi feita com a utilização da correlação de Pearson e test t de student para grupos normais, adotando-se nível de significância de $p<0,05$.

\section{RESULTADOS}

O G1 possuía média de idade de 74,2ะ6,37 anos, e o G2, 68,3ะ6,09 anos. Em relação à percepção de saúde, $10 \%$ das idosas do $\mathrm{G} 1$ consideraram a saúde como muito boa, $60 \%$ como boa, e $30 \%$ como ruim. Já no G2, $10 \%$ afirmaram que a saúde era muito boa, $80 \%$ disseram que era boa, e $10 \%$ responderam que era ruim.

A média e o desvio padrão da EMTI dos grupos estão apresentados na Tabela 1. O teste t evidenciou que não há diferença entre os grupos na EMTI.

Tabela 1 - Comparação do equilíbrio das idosas avaliado pela Escala Motora para Terceira Idade (EMTI)

\begin{tabular}{lcccc}
\hline Variável & Grupo & Média & $\mathrm{dp}$ & $\boldsymbol{p}$ \\
\hline \multirow{2}{*}{ EMTI } & G1 & 37,20 & 22,2 & \\
& G2 & 58,80 & 38,9 & $\mathrm{p}>0,05$ \\
\hline
\end{tabular}

EMTI: Escala Motora para Terceira Idade; dp: desvio padrão; G1: grupo de idosas institucionalizadas; G2: grupo de idosas não institucionalizadas

Fonte: autoria própria.

Observou-se que a correlação entre qualidade de vida e equilíbrio no $\mathrm{G} 1(0,757)$ é forte, ao passo que no $\mathrm{G} 2(0,448)$ é moderada $(p<0,05)$.

\section{DISCUSSÃO}

Os principais achados deste estudo apontaram que não existe diferença no equilíbrio e qualidade de vida de idosas institucionalizadas e não institucionalizadas; porém, vale lembrar que a amostra é pequena e de uma única região do país.

Quando indagadas sobre a autopercepção de saúde, apenas $10 \%$ das idosas (em ambos os grupos) relataram 
ser muito boa. Sabe-se que a Organização Mundial de Saúde (OMS, 2012) considera saúde como "Estado de completo bem-estar físico, mental e social e não apenas a ausência de doença". Porém, pessoas que passam por processo de institucionalização possuem maiores chances de desencadear distúrbios nesses parâmetros, como o surgimento da depressão. A autopercepção da saúde é importante na vida de um idoso, mesmo que ele apresente algumas doenças (JÓIA; RUIZ; DONALÍSIO, 2008).

É preciso lembrar que as ILPI prestam cuidados e assistência à saúde de idosos mais debilitados, porém acabam isolando-os do convívio em sociedade, limitando suas possibilidades de convivência e aprendizagem. Contudo, elas representam ainda uma das únicas alternativas viáveis que preservam o mecanismo de sobrevivência perante as dificuldades socioeconômicas, afetivas e familiares. A internação do idoso em ILPI gera deterioração da capacidade funcional e autoestima, restringindo a realização de tarefas que antes da internação eram rotineiras (ARAUJO; COUTINHO; SANTOS, 2006; PIZARRO, 2004; SILVESTRE; COSTA NETO, 2003).

Quando se trata do equilíbrio postural em idosas institucionalizadas, há diminuição dele, com maior risco de quedas, sequelas e piora da qualidade de vida principalmente nos quesitos relacionados a aspectos emocionais e sociais, vitalidade e dor (CARVALHO et al., 2013; LEGUISAMO et al., 2016; TRIBESS; VIRTUOSO JUNIOR, 2005).

Os achados desta pesquisa evidenciaram que as idosas institucionalizadas possuem o mesmo equilíbrio corporal que as não institucionalizadas. Isso pode ser justificado pela pequena amostra utilizada para o estudo, assim como fatores intrínsecos de cada idosa, como força muscular e flexibilidade. No trabalho desenvolvido por Borges et al. (2013), ao se avaliar o equilíbrio funcional, idosas institucionalizadas alcançaram uma pontuação média inferior à das idosas da comunidade, além de apresentar maior tendência a quedas e risco à depressão. Tal constatação pode ser consequência tanto do declínio das funções fisiológicas quanto do ambiente em que essas pessoas vivem. Porém, não foi isso o encontrado na presente pesquisa.

Quanto maior for o tempo de institucionalização, mais fragilizado ficará o idoso. Isso acontece após mudança de ambiente, e, sendo assim, há alteração da função psicológica, cognitiva e funcional. As consequências disso seriam comprometimento à saúde e qualidade de vida, aumento dos riscos de quedas, isolamento social e perda da autonomia, além do déficit de capacidade funcional (CARVALHO et al., 2013; FERREIRA; YOSHITOME, 2010; LEGUISAMO et al., 2016).

É importante lembrar que as idosas do $\mathrm{G} 2$ eram frequentadoras de ATI, ou seja, um local de prática de atividade física. Essa rotina poderia minimizar as alterações do aparelho locomotor geradas em decorrência do envelhecimento, além de reduzir a fragilidade óssea, dores articulares e decréscimos das funções, melhorando assim o equilíbrio postural. Principalmente o exercício físico, além de auxiliar na melhora da qualidade de vida de idosos, é uma estratégia para proporcionar bem-estar físico e psicológico, fazendo com que eles se sintam bem e mantenham o interesse na prática (PEDRINELLI; GARCEZ-LEME; NOBRE, 2009; SILVA et al., 2011; TEIXEIRA, 2007).

Nossos resultados apontaram também que existe uma relação significativa entre equilíbrio corporal e qualidade de vida em idosas, porém tal relação foi mais forte nas idosas institucionalizadas do que nas não institucionalizadas. Sabe-se que o déficit de equilíbrio pode gerar o maior risco de quedas e o medo de cair, o que pode ser um fator interveniente na qualidade de vida dessas pessoas (MACIEL; GUERRA, 2005).

Mesmo diante dos achados desta pesquisa, algumas limitações merecem destaque. Trata-se de um estudo transversal, o que impede inferir causalidade entre as variáveis. A amostra é pequena e por conveniência, além de ser composta de idosas de um único município do Brasil, não tornando possível generalizar os dados para outras populações com características semelhantes. Fazem-se, então, necessárias mais estratégias de promoção da saúde do idoso, institucionalizada ou não, referente à prática de exercícios físicos e aumento do nível de atividade física, a fim de incrementar ainda mais o equilíbrio dessas pessoas, com consequente melhora na qualidade de vida de forma geral.

\section{CONCLUSÃO}

Conclui-se, neste estudo, que não há diferença no equilíbrio entre idosos institucionalizados e não institucionalizados. Porém, a relação entre essa variável e a qualidade de vida é significativa e forte entre os institucionalizados, e moderada, entre os não institucionalizados.

Como implicações práticas, aponta-se a necessidade da prática de exercícios físicos como forma de melhora do equilíbrio e qualidade de vida desse público. Além de propiciar o aumento de força, a atividade elevaria o nível de equilíbrio e proporcionaria melhor qualidade de vida.

\section{REFERÊNCIAS}

ALLENDORF, D. B. et al. Idosos praticantes de treinamento resistido apresentam melhor mobilidade do que idosos fisicamente ativos não praticantes. Rev. Bras. Ciênc. Mov., São Paulo, v. 24, n. 1, p. 134-144, 2016.

ANTES, D. L. et al. Mortalidade por queda em idosos: estudo de série temporal. Rev. Bras. Geriatr. Gerontol., Rio de Janeiro, v. 18, n. 4, p. 767-778, 2015.

ARAUJO, L. F.; COUTINHO, M. P. L.; SANTOS, M. F. S. O idoso nas instituições gerontológicas: um estudo na perspectiva das representações sociais. Psicol. Soc., São Paulo, v. 18, n. 2, p. 89-98, 2006.

BRUCKI, S. M. et al. Sugestões para o uso do miniexame do estado mental no Brasil. Arq. Neuropsiquiatr., São Paulo, v. 61, n. 3-B, p. 777-781, 2003.

BORGES, M. G. S. et al. Comparação do equilíbrio, depressão e cognição entre idosas institucionalizadas e não institucionalizadas. Revista CEFAC, São Paulo, v. 15, n. 5, p. 1073-1079, 2013. 
CAMELIER, A. A. Avaliação da qualidade de vida relacionada à saúde em pacientes com DPOC: estudo de base populacional com o SF12 na cidade de São Paulo-SP. 2004. Tese (Doutorado em Ciências), Universidade Federal do Estado de São Paulo, São Paulo, 2004.

CARVALHO, E. et al. Relação entre as quedas, o equilíbrio funcional e a qualidade de vida em idosos. Ensaios e Ciência: Ciências Biológicas, Agrárias e da Saúde, v. 17, n. 1, p. 43-52, 2013.

FERREIRA, D. C. O.; YOSHITOME, A. Y. Prevalência e caraterísticas das quedas de idosos institucionalizados. Rev. Bras. Enferm., Brasília, v. 63, n. 6, p. 991-997, 2010.

FOLSTEIN, M. F.; FOLSTEIN, S. E.; MCHUGH, P. R. Mini mental State: a practical method for grading the cognitive state of patients for clinician. J. Psychiatr. Res., Oxford, v. 12, n. 1, p. 189-198, 1975.

FREITAS, A. P. et al. Relação da qualidade de vida com o estado nutricional de idosos. Rev. Bras. Qual. Vida, Ponta Grossa, v. 9, n. 1, p. 30-44, 2017.

JÓIA, L. C.; RUIZ, T.; DONALÍSIO, M. R. Grau de satisfação com a saúde entre idosos do Município de Botucatu, Estado de São Paulo, Brasil. Epidemiologia e Serviços de Saúde, Brasília, v. 17, n. 3, p. 187-194, 2008.

LEGUISAMO, C. P. et al. Efeito de um programa de exercícios físicos no equilíbrio e risco de quedas em idosos institucionalizados: ensaio clínico randomizado. Rev. Bras. Geriat. Gerontol., Rio de janeiro, v. 19, n. 3, p. 473-482, 2016.

MACIEL, A. C. C.; GUERRA, R. O. Prevalência e fatores associados ao déficit de equilíbrio em idosos. Rev. Bras. Ciênc. Mov., São Paulo, v. 13, n. 1, p. 37-44, 2005.

OMS - Organização Mundial de Saúde. Definition of an older or elderly person. Geneva, 2012. Disponível em: Acesso em: http://www.who.int/ healthinfo/survey/ageingdefnolder/en/index.html 01 Ago 2018.

PEDRINELLI, A.; GARCEZ-LEME, L. E.; NOBRE, R. S. A. O efeito da atividade física no aparelho locomotor do idoso. Revista Brasileira de Ortopedia, v. 44 , n. 2, p. 96-101, 2009.

PIZARRO, R. A. D. S. A importância da atuação do profissional enfermeiro na qualidade de vida dos idosos institucionalizados: uma avaliação qualitativa nas casas de repouso da cidade de São Paulo. 2004. Dissertação (Mestrado em Engenharia de Produção), Universidade Federal de Santa Catarina, Florianópolis, 2004.

PUCCI, G. C. M. F. et al. Association between physical activity and quality of life in adults. Rev. Saúde Pública, São Paulo, v. 46, n. 1, p. 166-179, 2012.

ROSA NETO, F. Manual de avaliação motora. Porto Alegre: Artmed, 2002.

SILVA, J. L. et al. Fatores associados à desnutrição em idosos institucionalizados. Rev. Bras. Geriatr. Gerontol., Rio de Janeiro, v. 18, n. 2, p. 443-451, 2015.

SILVA, P. et al. Cambios en la percepción del cuerpo de mujeres de edad avanzada, a través de un programa de actividad física. Eur. J. Hum. Mov., n. 26, p. 55-65, 2011.

SILVESTRE, J. A.; COSTA NETO, M. M. Abordagem do idoso em programas de saúde da família. Cad. Saúde Pública, Rio de Janeiro, v. 19, n. 3, p. 839-847, 2003.

TEIXEIRA, D. C. Efeitos de um programa de exercício físico para idosas sobre variáveis neuro-motoras, antropométricas e medo de cair. Rev. Bras. Educ. Fís Esp., São Paulo, v. 21, n. 2, p. 107-120, 2007.

TRIBESS, S.; VIRTUOSO JUNIOR, J. S. Prescrição de exercícios físicos para idosos. Revista Saúde.com., Jequié, v. 1, n. 2, p. 163-172, 2005.

Submetido em: 29/08/2019

Aceito em: 21/07/2020 